TRI-PP-00-20

\title{
Decays of the $B_{c}$ Meson in a Relativistic Quark-Meson Model
}

\author{
Matthew A. Nobes \\ Department of Physics, Simon Fraser University \\ Burnaby, BC, Canada V5A 1S6 \\ and \\ R. M. Woloshyn \\ TRIUMF, 4004 Wesbrook Mall, Vancouver, BC, Canada V6T 2A3
}

\begin{abstract}
The semileptonic decay form factors of the double heavy $B_{c}$ meson provide a unique opportunity to study the strong interactions between two heavy quarks. A fully relativistic model, with effective non-local quark-meson interactions, is used to compute semileptonic decay form factors, for both the $B_{c}$ and a wide range of other heavy-light mesons. Using these form factors predictions for decay rates and branching ratios are obtained. The results are compared to other theoretical approaches and, where available, to experimental results. In addition the radiative decay of $B_{c}^{*}$ is discussed.
\end{abstract}




\section{Introduction}

A primary goal in the study of semileptonic decays of heavy mesons is to extract the values of the CKM matrix elements. The great virtue of semileptonic decays is that the effects of the strong interaction can be separated from the effects of the weak interaction into a set of Lorentz invariant form factors [1]. Thus the theoretical problem associated with analysing semileptonic decays is essentially that of calculating the form factors.

The focus of this work is the decay of the $B_{c}$ meson (for a review of the properties of this system see [2]). This system is unique among mesons made up of heavy (charm or bottom) quarks, it is the only one which is stable with respect to strong and electromagnetic interactions. Therefore, the $B_{c}$ system is the only heavy meson for which form factors (albiet transition form factors rather than elastic) can be measured. These form factors then provide a unique probe of the dynamics of heavy quark systems.

There are many approaches to the calculation of decay form factors, for example, lattice QCD [3], QCD sum rules [4], and phenomological modelling [5]. In this work a particular model with an effective quark-meson coupling is adopted. There are many models of this type [6, 7, 8, 9, 10]. The one used here has its genesis in the QCD version of the Nambu-JonaLasinio model [11] extended to heavy quarks [6] and is most closely related to the model used recently by Ivanov and Santorelli in a their study of pseudoscalar meson decays [12].

The advantage of this approach is that it is fully relativistic and very versatile. Quarks and mesons for all masses are treated within the same framework. For light quarks the model has the features of spontaneous chiral symmetry breaking and in the single heavy quark limit the form factor constraints of heavy quark symmetry are obtained.

Our work differs from Ivanov and Santorelli in the choice of the quarkmeson vertex function and in the way that parameters are fixed. A number of heavy mesons decays not calculated in Ref.[12] are treated here. The 
main new results are the extension of the model to include doubly heavy mesons, the calculation of $B_{c}$ semileptonic decays and the electromagnetic vector to pseudoscalar transitions.

This paper is organized as follows: the next section introduces the model, discusses the general method of calculation, and fits the models free parameters. Sect. 3 presents the calculation of the form factors and decay rates for the semileptonic decays of a wide varity of heavy-light pseudoscalar mesons. These calculations are compared with both measured results, and other theoretical approaches. Sect. 4 presents the same set of calculations for the eight primary semileptonic decays of the $B_{c}$ meson. The predictions are compared with other theoretical work, in order to highlight the differences that exist between various approaches. Sect. 5 briefly discusses the electromagnetic decays $V \rightarrow P+\gamma$ for a number of vector mesons, including the $B_{c}^{*}$. Sect. 6 gives conclusions and directions for future work.

\section{Quark-Meson Coupling}

The particular quark-meson coupling used in this work is based on an effective Lagrangian which models the interaction between mesons and quarks with a non-local interaction vertex [6, 12]. The interaction Lagrangian has the form

$$
\begin{aligned}
L_{\text {int }}(x)= & g_{M} M(x) \int d x_{1} d x_{2} \delta\left(x-\left(\frac{1}{2} \frac{m_{1} x_{1}+m_{1} x_{2}}{m_{1}+m_{2}}\right)\right) \times \\
& f\left[\left(x_{1}-x_{2}\right)^{2}\right] \bar{q}_{1}\left(x_{1}\right) \Gamma_{M} q_{2}\left(x_{2}\right),
\end{aligned}
$$

where $\Gamma_{M}$ is the Dirac matrix appropriate to the meson field M, $f\left[\left(x_{1}-x_{2}\right)^{2}\right]$ is a non-local vertex function, which simulates the finite size of the meson, and $q_{1}$ and $q_{2}$ are the quark fields. A condition imposed on the vertex function is that it should render all loop diagrams UV finite. The coupling constant $g_{M}$ is determined by the compositeness condition, which is the 
requirement that the renormalization constant of the meson fields be zero, i.e.

$$
Z_{M}=1-\left.\frac{g_{M}^{2}}{2} \frac{d \Pi_{M}\left(p^{2}\right)}{d p^{2}}\right|_{p^{2}=M_{M}^{2}}=0 .
$$

Here $\Pi_{M}\left(p^{2}\right)$ is the self energy of the meson field, given by

$$
\begin{aligned}
\Pi_{M}\left(p^{2}\right)= & 2 N_{c} \int \frac{d^{4} k}{(2 \pi)^{4} i} f^{2}\left(Q^{2}\right) \\
& \operatorname{tr}\left\{\Gamma_{M} \frac{1}{m_{1}-(\not k+\not p)} \Gamma_{M} \frac{1}{m_{2}-\not k}\right\},
\end{aligned}
$$

where $m_{1}$ and $m_{2}$ are the masses of the quarks in the loop and $Q$ is a relative momentum chosen to be $Q=k+\alpha p$ with $\alpha=\frac{m_{2}}{m_{1}+m_{2}}$.

The constituent quark masses in (3) are free parameters. As well, the vertex function will contain a free parameter which reflects the size of the meson. These parameters will be different for the different mesons.

The use of free constituent quark propagators in expressions like (3) can lead to a problem which reflects the lack of quark confinement in the model. If the meson mass $M_{M}$ is greater than the sum of its constituent quark masses loop integrals will develop imaginary parts. This indicates a nonzero amplitude for the creation of a free quark-antiquark pair. There have been some various attempts to obviate this problem within quark-meson effective theories [7, 8, 9, 10]. Here we adopt the approach of Ref. [12] and use free propagators. The constituent quark masses are then fit to allow for the inclusion of as many mesons as possible.

In order to carry out calculations a choice must be made for the vertex function $f\left(q^{2}\right)$. The function that was used in this analysis was the dipole

$$
f\left(Q^{2}\right)=\frac{\Lambda^{4}}{\left[\Lambda^{2}-Q^{2}\right]^{2}} .
$$

This choice was made for two reasons; first the form of the dipole vertex function is the same as a propagator, allowing standard Feynman parameter techniques to be used in evaluating loop integrals. Second, the vector 
decay constant $f_{V}$ would diverge if only a monopole vertex function was used. Since one of the primary criteria for the vertex functions is that they should render all diagrams UV finite, a function with UV fall-off as least as fast as a dipole is needed. The parameter $\Lambda$ characterizes the finite size of the meson, and will be different for different mesons. To account for this the various values of $\Lambda$ will be distinguished by subscripts which reflect either the meson type or the quark content, e.g. $\Lambda_{B_{c}}$ and $\Lambda_{b c}$ will be used interchangeably. Further, in expressions involving the vertex form factor, the same comvention will be used. Note that the calculations of Ref. 12] used a Gaussian vertex function so that the parameters used there can not be compared directly with ours.

The parameters of the model were fit to the leptonic decay constants, $f_{P}$ and $f_{V}$. These quantities are defined by

$$
\begin{aligned}
\left\langle 0\left|-i \gamma^{\mu} \gamma^{5}\right| P\right\rangle & =i f_{P} p^{\mu} \\
\left\langle 0\left|i \gamma^{\mu}\right| V, \epsilon\right\rangle & =M_{V}^{2} f_{V} \epsilon^{\mu}
\end{aligned}
$$

where $M_{V}$ is the vector meson mass. The pseudoscalar decay constant is given by the one-loop expression

$$
\begin{aligned}
f_{P} p^{\mu}= & N_{c} \int \frac{d^{4} k}{(2 \pi)^{4} i} g_{P} f\left(Q^{2}\right) \\
& \frac{\operatorname{tr}\left\{-\gamma^{\mu} \gamma^{5}\left[m_{1}+\not k+\not p\right] \gamma^{5}\left[m_{2}+\not k\right]\right\}}{\left[m_{1}^{2}-(k+p)^{2}\right]\left[m_{2}^{2}-k^{2}\right]} .
\end{aligned}
$$

Here $m_{1}$ and $m_{2}$ refer to the masses of the quarks in the loop, this convention will be used throughout this paper. Using the dipole vertex function, combining the denominators using Feynman parameters, and performing the integration over $\mathrm{k}$ yields

$$
f_{P}=g_{P} \frac{3 \Lambda_{P}^{4}}{4 \pi^{2}} \int D \vec{x} \frac{x_{1}\left[m_{2}(1-\sigma)+m_{1} \sigma\right]}{\Delta^{2}}
$$

with

$$
\sigma=\alpha x_{1}+x_{2}
$$




$$
\begin{aligned}
\eta & =\alpha^{2} x_{1}+x_{2}, \\
\Delta & =\Lambda_{P}^{2} x_{1}+m_{1}^{2} x_{2}+m_{2}^{2} x_{3}+\left(\sigma^{2}-\eta\right) M_{P}^{2}, \\
\ell & =k+\sigma p \\
\int D \vec{x} & =\int_{0}^{1}\left(\prod_{i=1}^{3} d x_{i}\right) \delta\left(\sum_{i=1}^{3} x_{i}-1\right),
\end{aligned}
$$

where $M_{P}$ is the mass of the pseudoscalar meson. Likewise the expression for the vector decay constant is

$$
f_{V}=g_{V} \frac{3 \Lambda_{V}^{4}}{4 \pi^{2} M_{V}^{2}} \int D \vec{x} x_{1} \frac{m_{1} m_{2}+\Delta+\sigma(1-\sigma) M_{V}^{2}}{\Delta^{2}}
$$

where the same defintions have been used, with the obvious change of $M_{P}$ to $M_{V}$ and $\Lambda_{P}$ to $\Lambda_{V}$ in the expression for $\Delta$.

To compute the coupling constants $g_{P}$ and $g_{V}$, the self energies and their derivatives must be computed. Then the compositeness condition (2) can be used to find the couplings. The self energy for a pseudoscalar meson is given by

$$
\Pi_{P}\left(p^{2}\right)=\frac{3 \Lambda_{P}^{8}}{2 \pi^{2}} \int D \vec{x} x_{1}^{3} \frac{m_{1} m_{2}+p^{2} \sigma(1-\sigma)+\frac{2}{3} \bar{\Delta}}{\bar{\Delta}^{4}}
$$

where $\bar{\Delta}=\Lambda_{P}^{2} x_{1}+m_{1}^{2} x_{2}+m_{2}^{2} x_{3}+\left(\sigma^{2}-\eta\right) p^{2}$ and all the other quantities are the same as the ones defined above. The self energy for a pseudoscalar meson is given by the tensor $\Pi_{V}^{\mu \nu}$ which can be expressed as

$$
\Pi_{V}^{\mu \nu}(p)=\Pi_{V}\left(p^{2}\right) g_{\mu \nu}+\bar{\Pi}_{V}\left(p^{2}\right) \frac{p_{\mu} p_{\nu}}{p^{2}} .
$$

Unfortunately $\Pi_{V} \neq \bar{\Pi}_{V}$, so this does not have the proper structure for a vector propagator. This problem was solved (following [13]) by simply dropping the $\bar{\Pi}_{V}$ term, which would cancel out of any calculation of a physical process at one-loop order ( $\operatorname{since} \epsilon \cdot p=0$ ). The relevant part of the vector meson self energy is given by

$$
\Pi_{V}\left(p^{2}\right)=\frac{3 \Lambda_{V}^{8}}{2 \pi^{2}} \int D \vec{x} x_{1}^{3} \frac{m_{1} m_{2}+\frac{1}{3} \bar{\Delta}+\sigma(1-\sigma) p^{2}}{\bar{\Delta}^{4}},
$$


where all the quantities appearing have been defined previously.

The free parameters of the model are fit to the six values of $f_{P}$ and the measured values $f_{J / \psi}=0.1309$ and $f_{\Upsilon}=0.075012$ [14]. These data, which are displayed in Table 1, fix eight free parameters. In order to reduce the number of free parameters to match the available data the value of the strange quark mass was fixed at $450 \mathrm{MeV}$ and the vertex parameter for a vertex containing only $\mathrm{u}$ and d quarks $\Lambda_{\pi}$ was taken (following [6]) to be 1 $\mathrm{GeV}$. In addition the following further simplifying assumptions were made

$$
\begin{aligned}
& \Lambda_{u s}=\Lambda_{d s}=\Lambda_{s s}=\Lambda_{K}, \\
& \Lambda_{u c}=\Lambda_{d c}=\Lambda_{s c}=\Lambda_{D}, \\
& \Lambda_{u b}=\Lambda_{d b}=\Lambda_{s b}=\Lambda_{B} .
\end{aligned}
$$

This leaves the following parameters to be fit, $m_{q}, m_{c}, m_{b}, \Lambda_{K}, \Lambda_{D}, \Lambda_{B}$, $\Lambda_{c c}, \Lambda_{b b}$, and $\Lambda_{b c}$. The parameter $\Lambda_{b c}$ could only be fit to a value for $f_{B_{c}}$ which is not in the values listed in Table 1, hence it is retained as a free parameter, leaving eight to be fit. The fit to the remaining eight parameters is given by (all values in $\mathrm{MeV}$ )

$$
\begin{aligned}
m_{u, d} & =245, \\
m_{c} & =1800, \\
m_{b} & =5100, \\
\Lambda_{K} & =1225, \\
\Lambda_{D} & =1350, \\
\Lambda_{B} & =1500, \\
\Lambda_{c c} & =1420, \\
\Lambda_{b b} & =2900 .
\end{aligned}
$$

The values for the self energies, coupling constants, and leptonic decay constants arising from these parameters are displayed in Tables 2 and 3 . 
In order to fix $\Lambda_{b c}$ a value of $f_{B_{c}}$ must be given. There is no experimental value for this quantity and theoretical estimates tend to fall in the range $400 \mathrm{MeV} \lesssim f_{B_{c}} \lesssim 500 \mathrm{MeV}$ (see, for example, [2, 16, 17, 18, 19]). A further complication is that the mass of $M_{B_{c}}$ is also not yet known very well. The current measurement [20] is $M_{B_{c}}^{C D F}=6.4 \pm 0.39$ (stat) \pm 0.13 (sys) $\frac{\mathrm{GeV}}{c^{2}}$, which comes from the few confirmed $B_{c}$ events at the Tevatron. Theoretical results tend to lie within this range, so following the potential model prediction of [2] the mass of the $B_{c}$ was chosen to be $6.25 \mathrm{GeV}$.

One general argument guides the selection of $\Lambda_{b c}$, it should lie between $\Lambda_{B}$ and $\Lambda_{b b}$. With this in mind, and using the value for $M_{B_{c}}$ above, a number of values of $\Lambda_{b c}$ were tried, spanning the possible range. Fig. 1 1 shows the value of $f_{B_{c}}$ as a function of $\Lambda_{b c}$. The value selected selected for use in this work was $\Lambda_{b c}=2.3 \mathrm{GeV}$, which gives $f_{B_{c}}=450 \mathrm{MeV}$, a value in the middle of the range of the theoretical predictions.

\section{Semileptonic Decays of $K, D$, and $B$ Mesons}

The model used in this work is phenomenological but having fixed its parameters, the results for semileptonic decays are predictions. Before proceeding to decays of $B_{c}$ it is important to test the model against experimental results where they are available. Therefore several semileptonic decays of $K, D$, and $B$ meson are calculated. The formalism for these calculations, presented in this section, extends directly also to the calculation of $B_{c}$ decay.

Some of the decays considered here have already been treated by Ivanov and Santorelli [12]. However, that work does not demonstrate the full applicablity of the approach. Apart from decays to light vector mesons, the model is capable of treating virtually any semileptonic decay (with the restriction that a value for the meson mass must be supplied as input).

The amplitude $A$ for a semileptonic decay is given by,

$$
A=\frac{G_{F}}{\sqrt{2}} V_{Q Q^{\prime}} L_{\mu} H^{\mu} .
$$


Here $G_{F}$ is the Fermi constant, $V_{Q Q^{\prime}}$ is the relevant CKM matrix element, $L_{\mu}$ is the lepton current

$$
L_{\mu}=\bar{u}_{\nu_{\ell}} \gamma_{\mu}\left(1-\gamma^{5}\right) v_{\ell}
$$

and $H^{\mu}$ is the hadron current

$$
H^{\mu}=\left\langle k, \epsilon\left|\left(V^{\mu}-A^{\mu}\right)\right| P\right\rangle,
$$

where $P$ is the momentum of the parent meson, $k$ is the momentum of the daughter meson, and $\epsilon$ is the polarization, if the daughter meson is a vector. The two currents in (13) are the vector $V^{\mu}$ and axial $A^{\mu}$. If the final state is a pseudoscalar the hadron current can be decomposed as follows,

$$
\begin{aligned}
\left\langle k\left|A^{\mu}\right| P\right\rangle & =0, \\
\left\langle k\left|V^{\mu}\right| P\right\rangle & =f_{+}\left(q^{2}\right)(P+k)^{\mu}+f_{-}\left(q^{2}\right)(P-k)^{\mu},
\end{aligned}
$$

where $f_{+}\left(q^{2}\right)$ and $f_{-}\left(q^{2}\right)$ are Lorentz invariant form factors. Likewise, if the final state is a vector meson,

$$
\begin{aligned}
\left\langle k, \epsilon\left|A^{\mu}\right| P\right\rangle= & f\left(q^{2}\right) \epsilon^{* \mu}+a_{+}\left(q^{2}\right)\left(\epsilon^{*} \cdot P\right)(P+k)^{\mu}+ \\
& a_{-}\left(q^{2}\right)\left(\epsilon^{*} \cdot P\right)(P-k)^{\mu}, \\
\left\langle k, \epsilon\left|V^{\mu}\right| P\right\rangle= & i g\left(q^{2}\right) \epsilon^{\mu \nu \rho \sigma} \epsilon_{\nu}^{*}(P+k)_{\rho}(P-k)_{\sigma},
\end{aligned}
$$

where the form factors are $g, f, a_{+}$, and $a_{-}$. In each of these expressions $q=(P-k)$ is the momentum transfer.

For a decay to a pseudoscalar meson (with mass denoted by $M_{P^{\prime}}$ ) the differential decay rate can be reduced to [1]

$$
\frac{d \Gamma}{d q}=\frac{G_{F}^{2}\left|V_{Q Q^{\prime}}\right|^{2} M_{P}^{2} K^{3}}{24 \pi^{3}}\left|f_{+}\left(q^{2}\right)\right|^{2} .
$$

where,

$$
K=\frac{M_{P}}{2} \sqrt{\left[1-\frac{M_{P^{\prime}}^{2}}{M_{P}^{2}}-y\right]^{2}-4 \frac{M_{P^{\prime}}^{2}}{M_{P}^{2}} y}
$$


The lepton spectrum is given by,

$$
\begin{aligned}
\frac{d \Gamma}{d x}= & \frac{G_{F}^{2}\left|V_{Q Q^{\prime}}\right|^{2} M_{P}^{5}}{16 \pi^{3}}(1-2 x) \\
& \int_{0}^{y_{\max }(x)}\left(\left[y_{\max }(x)-y\right]\left|f_{+}\left(q^{2}\right)\right|^{2}\right) d y,
\end{aligned}
$$

where $y_{\max }(x)=\frac{4 x\left(x_{\max }-x\right)}{1-2 x}$ with $x_{\max }=\frac{M_{P}^{2}-M_{P^{\prime}}^{2}}{2 M_{P}^{2}}$. If the final state is a vector meson (with mass $M_{V}$ ) the corresponding differential decay rate is,

$$
\frac{d \Gamma}{d y}=\frac{G_{F}^{2}\left|V_{Q Q^{\prime}}\right|^{2} K M_{P}^{2} y}{96 \pi^{3}}\left(\left|\bar{H}_{+}\right|^{2}+\left|\bar{H}_{-}\right|^{2}+\left|\bar{H}_{0}\right|^{2}\right),
$$

where

$$
\begin{aligned}
\bar{H}_{ \pm} & =f\left(q^{2}\right) \mp 2 M_{P} K g\left(q^{2}\right), \\
\bar{H}_{0} & =\left[\frac{M_{P}}{2 M_{V} \sqrt{y}}\right]\left[\left(1-\frac{M_{V}^{2}}{M_{P}^{2}}-y\right) f\left(q^{2}\right)+4 K^{2} a_{+}\left(q^{2}\right)\right],
\end{aligned}
$$

and the final mass $M_{V}$ should be subsititued for $M_{P^{\prime}}$ in (15). The expression for the lepton spectrum is given by

$$
\begin{aligned}
\frac{d \Gamma}{d x}= & \frac{G_{F}^{2}\left|V_{Q Q^{\prime}}\right|^{2} M_{P}^{5}}{32 \pi^{3}} \int_{0}^{y_{\max }(x)}\left\{\frac{\alpha(y)}{M_{P}^{2}} y+\right. \\
& 2(1-2 x)\left[y_{\max }(x)-y\right] \beta_{++}(y)+ \\
& \left.\gamma(y) y\left[2 x_{\max }-4 x+y\right]\right\},
\end{aligned}
$$

where the following definitions were made

$$
\begin{aligned}
\alpha\left(q^{2}\right)= & \left|f\left(q^{2}\right)\right|^{2}+\lambda\left|g\left(q^{2}\right)\right|^{2}, \\
\gamma\left(q^{2}\right)= & 2 f\left(q^{2}\right) g\left(q^{2}\right), \\
\beta_{++}\left(q^{2}\right)= & \frac{1}{4 M_{V}}\left\{\left|f\left(q^{2}\right)\right|^{2}+\lambda\left|a_{+}\left(q^{2}\right)\right|^{2}-4 M_{V}^{2} q^{2}\left|g\left(q^{2}\right)\right|^{2}\right. \\
& \left.+2\left(M_{P}^{2}-M_{V}^{2}-q^{2}\right) f\left(q^{2}\right) a_{+}\left(q^{2}\right)\right\}, \\
\lambda\left(q^{2}\right)= & \left(M_{P}^{2}-M_{V}^{2}-q^{2}\right)^{2}-4 M_{V}^{2} q^{2}, \\
x_{\max }= & \frac{M_{P}^{2}-M_{V}^{2}}{2 M_{P}^{2}}
\end{aligned}
$$

Note that all of these expressions assume that lepton mass $m_{\ell}$ is zero. 
Our model can be used to calculate all of these form factors. In all the following expressions the inital meson is composed of quarks with masses $m_{1}$ and $m_{2}$ and the final meson is composed of quarks with masses $m_{3}$ and $m_{2}$ (i.e. $m_{1}$ is the mass of the quark which decays to a new quark with mass $m_{3}$, and $m_{2}$ is the mass of the spectator).

The form factors for decay to a pseudoscalar meson are

$$
\begin{aligned}
f_{+}\left(q^{2}\right)= & g_{P} g_{P^{\prime}} \frac{9 \Lambda_{P}^{4} \Lambda_{P^{\prime}}^{4}}{\pi^{2}} \\
& \int D \vec{x} x_{1} x_{2} \frac{\chi_{+}-\frac{3}{4} \bar{\Delta}\left(\sigma_{1}+\sigma_{2}\right)+\bar{\Delta}}{\bar{\Delta}^{5}}, \\
f_{-}\left(q^{2}\right)= & g_{P} g_{P^{\prime}} \frac{9 \Lambda_{P}^{4} \Lambda_{P^{\prime}}^{4}}{\pi^{2}} \\
& \int D \vec{x} x_{1} x_{2} \frac{\chi_{-}-\frac{3}{4} \bar{\Delta}\left(\sigma_{1}-\sigma_{2}\right)}{\bar{\Delta}^{5}} .
\end{aligned}
$$

The following definitions were made to simplify the expressions

$$
\begin{aligned}
& \int D \vec{x}=\int_{0}^{1}\left(\prod_{i=1}^{5} d x_{i}\right) \delta\left(\sum_{i=1}^{5} x_{i}-1\right), \\
& \Delta=\Lambda_{P^{\prime}}^{2} x_{1}+\Lambda_{P}^{2} x_{2}+m_{3}^{2} x_{3}+m_{1}^{2} x_{4}+m_{2} x_{5}, \\
& \mu_{i j}=\frac{m_{i}}{m_{i}+m_{j}} \\
& \sigma_{1,(2)}=x_{4,(3)}+\mu_{12,(23)} x_{2,(1)} \text {, } \\
& \eta_{1,(2)}=x_{4,(3)}+\mu_{12,(23)}^{2} x_{2,(1)} \text {, } \\
& \bar{\Delta}=\Delta+\left(\sigma_{1}^{2}-\eta_{1}+\sigma_{1} \sigma_{2}\right) M_{P}^{2} \\
& +\left(\sigma_{2}-\eta_{2}+\sigma_{1} \sigma_{2}\right) M_{P^{\prime}}^{2}-\sigma_{1} \sigma_{2} q^{2}, \\
& \kappa=m_{3}\left(m_{2}-m_{1}\right)+m_{1} m_{2}+\frac{1}{2}\left(M_{P}^{2}+M_{P^{\prime}}^{2}-q^{2}\right) \text {, } \\
& \epsilon=\left(\sigma_{1}+\sigma_{2}\right) \sigma_{1} M_{P}^{2}+\left(\sigma_{1}+\sigma_{2}\right) \sigma_{2} M_{P^{\prime}}^{2}-\sigma_{1} \sigma_{2} q^{2}, \\
& \zeta_{1}=m_{1} m_{2}-\left[\sigma_{1}\left(\sigma_{1}-1\right)+\sigma_{2}\left(\sigma_{1}-\frac{1}{2}\right)\right] M_{P}^{2} \\
& -\sigma_{2}\left(\sigma_{1}+\sigma_{2}-\frac{1}{2}\right) M_{P^{\prime}}^{2}+\sigma_{2}\left(\sigma_{1}-\frac{1}{2}\right) q^{2}, \\
& \zeta_{2}=m_{2} m_{3}-\sigma_{1}\left(\sigma_{1}+\sigma_{2}-\frac{1}{2}\right) M_{P}^{2}
\end{aligned}
$$




$$
\begin{aligned}
& -\left[\sigma_{2}\left(\sigma_{2}-1\right)+\sigma_{1}\left(\sigma_{2}-\frac{1}{2}\right)\right] M_{P^{\prime}}^{2} \\
& +\sigma_{1}\left(\sigma_{2}-\frac{1}{2}\right) q^{2}, \\
\chi_{ \pm}= & (\epsilon-\kappa)\left(\sigma_{1} \pm \sigma_{2}\right) \pm \zeta_{1}+\zeta_{2} .
\end{aligned}
$$

These definitions (in addition to $\alpha$ and $\mu$ ) will be used throughout the rest of this paper, with the obvious substitution of $M_{V}$ and $\Lambda_{V}$ for $M_{P^{\prime}}$ and $\Lambda_{P^{\prime}}$ when the final state is a vector meson.

The form factors for decays to vector mesons are given by

$$
\begin{gathered}
g\left(q^{2}\right)=g_{M_{P}} g_{M_{V}} \frac{9 \Lambda_{P}^{4} \Lambda_{V}^{4}}{\pi^{2}} \int D \vec{x} x_{1} x_{2} \\
\frac{\sigma_{2}\left(m_{2}-m_{3}\right)+\sigma_{1}\left(m_{2}-m_{1}\right)-m_{2}}{\bar{\Delta}^{5}}, \\
f\left(q^{2}\right)=g_{M_{P}} g_{M_{V}} \frac{18 \Lambda_{P}^{4} \Lambda_{V}^{4}}{\pi^{2}} \int D \vec{x} x_{1} x_{2} \times \\
\frac{1}{\bar{\Delta}^{5}}\left[m_{1} m_{2} m_{3}-\frac{1}{4}\left(m_{2}-m_{1}-2 m_{3}\right) \bar{\Delta}\right. \\
\left.+\left[\xi_{1}+\xi_{3}\right] M_{P}^{2}+\left[\xi_{2}+\xi_{3}\right] M_{V}^{2}-\xi_{3} q^{2}\right], \\
a_{ \pm}\left(q^{2}\right)=\frac{18 \Lambda_{P}^{4} \Lambda_{V}^{4}}{\pi^{2}} \int D \vec{x} x_{1} x_{2} \\
\frac{g_{M_{P}} g_{M_{V}}\left(\beta_{1} \pm \beta_{2} \mp \sigma_{2} m_{3}\right)}{\bar{\Delta}^{5}} .
\end{gathered}
$$

The following further definitions have been made,

$$
\begin{aligned}
\xi_{1}= & \sigma_{1}\left(m_{3}-m_{2}\right)\left(1-\sigma_{1}\right)-m_{1} \sigma_{1}^{2}, \\
\xi_{2}= & \sigma_{2}\left(m_{1}-m_{2}\right)\left(1-\sigma_{2}\right)-m_{3} \sigma_{2}^{2}, \\
\xi_{3}= & m_{2}\left[\frac{1}{2}-\frac{1}{2}\left(\sigma_{1}+\sigma_{2}\right)+\sigma_{1} \sigma_{2}\right]+m_{1} \sigma_{1}\left(\frac{1}{2}-\sigma_{2}\right) \\
& +m_{3} \sigma_{2}\left(\frac{1}{2}-\sigma_{1}\right), \\
\beta_{1}= & 2 \sigma_{1}\left[m_{1} \sigma_{1}+m_{2}\left(1-\sigma_{1}\right)\right], \\
\beta_{2}= & m_{2}\left(\sigma_{1}+\sigma_{2}-1-2 \sigma_{1} \sigma_{2}\right)-m_{1} \sigma_{1}\left(1-2 \sigma_{2}\right) .
\end{aligned}
$$


Excluding the $B_{c}$ decays a total of sixteen pseudoscalar to pseudoscalar decays were considered. Due to the difficulty with confinement the corresponding number of pseudoscalar to vector decays that could be treated was only four. Table 4 shows the predictions for the decay rates and branching ratios for all of the decays considered. The values of the CKM matrix elements, and the necessary lifetimes were taken from [14].

Many of the decay rates treated in this section have been measured, hence most of the predictions can be compared to observed quantities. Table 4 shows the predicted and measured results for the branching ratios. The experimental results are taken from [14] and the errors in the predictions represent the uncertainties in the CKM matrix elements. Overall, the agreement with experiment is reasonable which increases the level of confidence in the areas where direct comparison with experiment is not possible.

Table 5 shows values of $f_{+}(0)$ as computed in this work and in various other theoretical approaches. The other approaches are widely varied: [21] uses the ISGW model, [5] uses the WBS model, [22] gives results from a bag model, [23] uses a Dyson-Schwinger equation approach and [3] gives lattice QCD results. Of particular interest is the work of Ivanov et al. [24] which uses the quark confinement model. This quark-meson model is based on similar considerations to the model used in this work so its predictions should be close to ours.

As well, the decay $B \rightarrow D+\ell^{+}+\nu_{\ell}$ can be treated in a model independent way using the HQET. Ivanov et al. have shown in several papers [10, 12, 25] that quark-meson models of the type used here give the correct tree level HQET relations in the infinite mass limit. Nevertheless a direct check with finite quark mass is useful. The HQET gives the prediction 26]

$$
f_{+}^{H Q E T}\left(q_{\max }^{2}\right)=\frac{m_{B^{0}}+m_{D^{-}}}{2 \sqrt{m_{B^{0}} m_{D^{-}}}}=1.138,
$$

which compares well with our value $f_{+}\left(q_{\max }^{2}\right)=1.133$.

The most important comparison that can be made is with Ref. [12]. This paper uses a different vertex function to treat $\mathrm{B}$ and $\mathrm{D}$ decays. This serves 
as a check on the dependence of the model on the choice of vertex function. Apart from the case $B \rightarrow \pi+\ell+\nu$ agreement with [12] is very good. In addition [12] presents the values of $f_{+}\left(q^{2}\right)$ over the full range of $q^{2}$. Overall agreement is good between the two calculations, Fig. 2 illustrates the agreement in the case $D^{0} \rightarrow K^{-}+\ell^{+}+\nu_{\ell}$. Fig. 3 shows the case $B^{0} \rightarrow \pi^{-}+\ell^{+}+\nu_{\ell}$, for which the agreement is better over the whole range than indicated in Table 5 .

Due to lack of confinement very few pseudoscalar to vector decays can be calculated. Of the few decays treated in this work only the decay $B \rightarrow$ $D^{*}+\ell+\nu_{\ell}$ has been studied extensively. Table 6 compares our predictions with some other calculations. Overall the agreement is reasonable.

\section{Semileptonic Decays of the $B_{c}$ Meson}

The methods of the previous section can be directly applied to the semileptonic $B_{c}$ decays. Using the procdeure outline above, decay rates, lepton spectra, and branching ratios can be computed. In this work the lifetime of the $B_{c}$ was taken to be $0.5 \mathrm{ps}$, which agrees with the CDF value of $\tau_{B_{c}}^{C D F}=0.46_{-0.16}^{+0.18} \pm 0.03$ ps [20]. Table 7 shows $f_{+}(0), f_{+}\left(q_{\max }^{2}\right)$, the total decay rate $\Gamma$ and the branching ratio for the four pseudoscalar decays. For the decays to vector mesons, values of the form factors at $q^{2}=0$ as well as total decay rates and branching ratios are displayed in Tables 8 and 9 .

There are a number of other calculations of the semileptonic decays of $B_{c}$. A comparison of some results for the dominant decay modes is given in Table 10. In contrast to the situation in Sect. 3 where our quark-meson model predictions, for the most part, agreed with other models and the various other models agreed with each other, there are substantial differences between calculations of $B_{c}$ decays. The clearest examples of this are the predictions for the decays to the $B_{s}^{*}$ and $J / \psi$. These two decays are expected to be the most important semileptonic decay channels However there is disagreement not only over the values of the branching ratios but also as to 
which decay will be favoured. For example, the quark-meson model used in this work predicts the decay to the $J / \psi$ to be slightly favoured over the decay to the $B_{s}^{*}$ while the heavy quark approach used in [28] and [30] predicts the decay to $B_{s}^{*}$ to dominate. This divergence of predictions may be expected; the heavy-heavy quark content of the $B_{c}$ poses a challenge for models. Light-quark mesons may be constrained by chiral symmetry and heavy-light mesons by heavy quark symmetry. On the other hand the physics of heavy-heavy systems is less constrained by symmetries so extending models into this domain provides a severe test.

\section{$5 \quad$ Electromagnetic Decays $V \rightarrow P+\gamma$}

In addition to semileptonic decays the electromagnetic decays of vector mesons can be treated within our effective quark-meson coupling model. Since the amplitude involves the matrix element $\left\langle V\left|V^{\mu}\right| P\right\rangle$ it is clear this process will be related to the form factor $g\left(q^{2}\right)$. The the amplitude for this process is

$$
A=-2 e \epsilon^{\mu \nu \alpha \beta} \tilde{\epsilon}_{\mu} \epsilon_{\nu} p_{V \alpha} p_{P \beta}\left[Q_{1} g_{1}(0)+Q_{2} g_{2}(0)\right]
$$

where $Q_{1,(2)}$ is the charge of $q_{1,(2)}$, and $p_{P, V}$ are the momenta of the pseudoscalar and vector mesons. The functions $g_{i}(0)$ are the form factors given by (21), with the appropriate masses inserted, and with $q^{2}=0$. The appropriate masses in these functions are given by the interchange of $M_{P}$ and $M_{V}$ and the subscript which denotes which of the quark lines the gauge field is coupled to (i.e. for $g_{1}(0)$ the appropriate expression sets $m_{3}=m_{1}$ ). Defining $g_{V P \gamma}=2\left[Q_{1} g_{1}+Q_{2} g_{2}\right]$, and summing over initial and final polarizations gives

$$
|A|^{2}=\frac{2 \alpha \pi}{3} M_{V}^{4}\left[1-\frac{M_{P}^{2}}{M_{V}^{2}}\right]^{2} g_{V P \gamma}^{2}
$$


where $\alpha=\frac{1}{137}$ is the fine structure constant. Standard techniques 31] yield the total rate

$$
\Gamma_{V P \gamma}=\frac{\alpha}{24} M_{V}^{3} g_{V P \gamma}^{2}\left(1-\frac{M_{P}^{2}}{M_{V}^{2}}\right)^{3} .
$$

Electromagetic decays have been the subject of several theoretical studies. As well the decay $J / \psi \rightarrow \eta_{c}+\gamma$ has been measured. Table 11 shows our predictions for $g_{V P \gamma}$ along with the single experimental result and the predictions of some other models. In [32] and [33] two different heavy quark approaches were used. The quark confinement model [13], which has some similarity to the quark-meson model used in this work, gives results which are quite close to ours.

There are measured branching ratios for the $D^{*}$ decays, however no lifetime measurement has been made. Therefore our predictions (which do not include the lifetime) cannot be compared directly with experiment. In order to obtain branching ratios a theoretical estimate of the lifetime must be used. The quark confinement model is the ideal choice, since its predictions are closest to our work. Using the results from [13] and our predictions for the total rates (obtained from (26)) the following branching ratios are obtained:

$$
\begin{aligned}
B R\left[\left(D^{*}\right)^{0} \rightarrow D^{0}+\gamma\right] & =33.0 \%, \\
B R\left[\left(D^{*}\right)^{+} \rightarrow D^{+}+\gamma\right] & =1.43 \% .
\end{aligned}
$$

These compare well with the experimental values 14]

$$
\begin{aligned}
B R_{\text {expt. }}\left[\left(D^{*}\right)^{0} \rightarrow D^{0}+\gamma\right] & =38.1 \% \\
B R_{\text {expt. }}\left[\left(D^{*}\right)^{+} \rightarrow D^{+}+\gamma\right] & =1.1 \%
\end{aligned}
$$

In order to treat the electromagnetic decay $B_{c}^{*} \rightarrow B_{c}+\gamma$ the mass of the $B_{c}^{*}$ meson must be specified. Theoretical estimates [2] indicate that the mass difference should be small; $M_{B_{c}^{*}}-M_{B_{c}}<100 \mathrm{MeV}$. To examine the effect of a small change in the $B_{c}^{*}$ mass, the self energy and coupling constant 
were calculated over a range of masses. These results were used to calculate $g_{B_{c}^{*} B_{c} \gamma}$ and are displayed in Table 12. The decay rate is shown in Fig. ․

The radiative decay of $B_{c}^{*}$ has not been studied extensively. A QCD sum rule approach [34], using $M_{B_{c}^{*}}=6.6 \mathrm{GeV}$ and $M_{B_{c}}=6.3 \mathrm{GeV}$, gives the result $g_{B_{c}^{*} B_{c} \gamma}^{S R}=0.270 \pm 0.095 \mathrm{GeV}^{-1}$. Using these masses our prediction is $g_{B_{c}^{*} B_{c} \gamma}=0.2196 \mathrm{GeV}^{-1}$. The two values are in agreement.

\section{Conclusion}

A Lagrangian which models mesons in terms of an effective non-local quarkmeson interaction vertex [6, 12 was extended in this paper to describe mesons such as $B_{c}^{*}$ composed of two heavy quarks. The model has the advantage of treating all quarks (heavy and light) on the same footing, thereby permitting a unified investigation of heavy $\rightarrow$ heavy, and heavy $\rightarrow$ light quark decays. The model does not provide a complete dynamical description of quark interactions, meson masses can not be calculated and must be introduced as input parameters. Quark masses and the parameters associated with the quark-meson vertex were determined by fitting the pseudoscalar and vector meson decay constants $f_{P}$ and $f_{V}$. Due to lack of confinement in this approach some light vector mesons had to be excluded from the analysis.

To both test the model, and demonstrate its versatility, a large number of semileptonic decays of $K, D$, and $B$ mesons were analysed. Agreement with measured results and other theoretical approaches was good.

The main focus of this work was on the analysis of the semileptonic decays of the doubly-heavy $B_{c}$ meson. The form factors characterizing the strong interactions of the $B_{c}$ system were computed over the entire available range of momentum transfer. Using these results, decay rates and branching ratios were computed for all eight decay going to mesonic ground states.

A comparison with some other approaches highlighted the significant differences among various model predictions concerning the $B_{c}$. On very 
general grounds one would expect the decays to $J / \psi$ and $\left(B_{s}^{*}\right)^{0}$ states to be the most important. However, there is no agreement from models which of the channels dominates and absolute rates differ by a factor of 3 to 4 . This is in contrast to the situation in $\mathrm{K}, \mathrm{D}$ and $\mathrm{B}$ meson decays where much smaller differences between different models are found.

As a further illustration of the versatility of the model, the electromagnetic decays $V \rightarrow P+\gamma$ were investigated. A reasonable description of $J / \psi$ and $D^{*}$ radiative decay was found and the rate for $B_{c}^{*} \rightarrow B_{c}+\gamma$ was calculated for a range of $B_{c}^{*}$ masses.

There is room for further analysis within the model considered in this work. Hadronic decays, such as $B_{c}^{+} \rightarrow J / \psi+\pi^{+}$can also be treated. A detailed analysis of all of these decays, combined with the results for the leptonic and semileptonic decays, could be used to make a prediction for the lifetime $\tau_{B_{c}}$. A further area that needs work is the difficulty with confinement. The natural solution to this problem appears to be provided by the quark confinement model [10]. Recently Ivanov et al. have proposed a modification of the quark confinement model which may aid its application to heavy mesons [35].

Acknowledgements. We would like to thank M.A. Ivanov and P. Santorelli for a helpful communication. This work was supported in part by the Natural Sciences and Engineering Research Council of Canada.

\section{References}

[1] Gilman F J and Singleton R L 1990 Phys. Rev. D 41142

[2] Gershtein S S, Kiselev V V, Likhoded A K, Tkabladze A V, Berezhnoy A V and A.I. Onishchenko "Theoretical Status of the $B_{c}$ Meson," hepph/9803433. 
[3] Flynn J M and Sachrajda C T "Heavy Quark Physics From Lattice QCD," hep-lat/9710057.

[4] Bagan E, Dosch H G, Gosdzinsky P, Narison S and Richard J M 1997 Z. Phys. C 6457

[5] Wirbel M, Stech B, and Bauer M 1985 Z. Phys. C 29637

[6] Anikin I V, Ivanov M A, Kulimanova N B and Lyubovitskij V E 1995 Z. Phys. C 65681

[7] Sutherland M, Holdom B, Jaimungal S and Lewis R 1995 Phys. Rev. D $\mathbf{5 1 5 0 5 3}$

[8] Blaschke D, Burau G, Volkov M K and Yudichev V L "NJL Model With Infrared Confinement," hep-ph/9812503.

[9] Deandrea A "A Constituent Quark-Meson Model for Heavy Meson Decays," hep-ph/9809393.

[10] Efimov G V and Ivanov M A 1993 The Quark Confinement Model of Hadrons (IOP Publishing, Bristol and Philadelphia)

[11] Nambu Y and Jona-Lasino G 1961 Phys. Rev. 122345

[12] Ivanov M A and Santorelli P 1999 Phys. Lett. B 456248

[13] Ivanov M A and Valit Y M 1995 Z. Phys. C 67633

[14] Particle Data Group:Caso C et al. 1998 Eur. Phys. Jour. C3 1

[15] Ryan S M and Simone J N 1998 Nuc. Phys. B (Proc. Suppl.) 63A-C 353

[16] Eichten E J and Quigg C 1994 Phys. Rev. D 495845

[17] Colangelo P and De Fazio F "Radiative Leptonic $B_{c}$ Decays," hepph/9904363. 
[18] Anisimov A Y, Kulikov P Y, Narodetskii I M and Ter-Martirosyan K A "Exclusive and Inclusive Decays of the $B_{c}$ Meson in the Light-Front ISGW Model," hep-ph/9809249.

[19] Jones B D and Woloshyn R M 1999 Phys. Rev. D 60014502

[20] Abe F et al, CDF Collaboration 1998 Phys. Rev. Lett. 812432

[21] Demchuk N B, Grach I L, Narodetskii I M and Simula S 1996 Phys. Atom. Nucl. 59, 2152

[22] Sadzikowski M 1995 Z. Phys. C 67129

[23] Ivanov M A, Kalinovsky Y L and C.D. Roberts C D 1999 Phys. Rev. D 60034018

[24] Ivanov M A, Mizutani T, and Valit Y M "Heavy-to-Light Form Factors in the Quark Model with Heavy Infrapropagators," hep-ph/9606404.

[25] Ivanov M A, Khomutenko O E, and Mizutani T 1992 Phys. Rev. D 46 3817

[26] Neubert M "B Decays and the Heavy Quark Expansion," hepph/9702375.

[27] Scora D and Isgur N 1989 Phys. Rev. D 401491

[28] Sanchis-Lozano M A 1995 Nucl. Phys. B 440251

[29] Kiselev V V, Likhoded A K, and Onishchenko A I "Semileptonic $B_{c^{-}}$ Meson Decays in Sum Rules of QCD and NRQCD," hep-ph/9905359.

[30] Colangelo P and De Fazio F "Using Heavy Quark Spin Symmetry in Semileptonic $B_{c}$ Decays," hep-ph/9909423.

[31] Peskin M E and Schroeder D V 1995 An Introduction to Quantum Field Theory (Addison-Wesley, New York) 
[32] Dosch H G and Narison S 1996 Phys. Lett. B 368163

[33] Colangelo P, De Fazio F and Nardulli F 1993 Phys. Lett. B 316555

[34] Aliev T M, Iltan E and Pak N K 1994 Phys. Lett. B 329123

[35] Ivanov M A, Santorelli P and Tancredi N "The Semileptonic Form Factors of B and D Mesons in the Quark Confinement Model," hepph/9905209. 
Table 1: Pseudoscalar Decay Constants

\begin{tabular}{|c|c|c|}
\hline Meson $(\mathrm{P})$ & $f_{P}(\mathrm{MeV})$ & Source \\
\hline \hline$\pi^{+}$ & $130.7 \pm 0.5$ & Expt. [14 \\
$K^{+}$ & $159.8 \pm 1.8$ & Expt. [14 \\
$D^{+}$ & $183_{-13-0-25}^{+12+41+9}$ & Lattice QCD 15 \\
$D_{s}^{+}$ & $229_{-11-0-19}^{+10+51+3}$ & Lattice QCD 15] \\
$B^{+}$ & $156_{-14-0-9}^{+12+29+9}$ & Lattice QCD 15 \\
$B_{s}^{0}$ & $177_{-12-0-11}^{+11+39+13}$ & Lattice QCD 15] \\
\hline
\end{tabular}

Table 2: Fitted Values of Pseudoscalar Meson Properties

\begin{tabular}{|c|c|c|c|}
\hline Meson & $\Pi\left(M^{2}\right)\left(\mathrm{GeV}^{2}\right)$ & $g_{P}$ & $f_{P}(\mathrm{MeV})$ \\
\hline \hline$\pi^{+}$ & 0.038588 & 5.14916 & 131.06 \\
$K^{+}$ & 0.068760 & 5.16139 & 160.85 \\
$D^{+}$ & 0.083122 & 6.26671 & 182.80 \\
$D_{s}^{+}$ & 0.087025 & 6.82039 & 223.81 \\
$B^{+}$ & 0.134842 & 6.44510 & 142.21 \\
$B_{s}^{0}$ & 0.136072 & 7.75408 & 187.42 \\
\hline
\end{tabular}

Table 3: Fitted Values of Vector Meson Properties

\begin{tabular}{|c|c|c|c|}
\hline Meson & $\Pi\left(M^{2}\right)\left(\mathrm{GeV}^{2}\right)$ & $g_{V}$ & $f_{V}$ \\
\hline \hline$J / \psi$ & 0.095419 & 8.81505 & 0.131227 \\
$\Upsilon$ & 0.621464 & 7.54393 & 0.074796 \\
\hline
\end{tabular}


Table 4: Predictions for Decay Rates and Branching Ratios

\begin{tabular}{|c|c|c|c|c|}
\hline Parent & Daughter & $\Gamma\left(\mathrm{ps}^{-1}\right)$ & $B R(\%)$ & $B R_{\text {expt. }}(\%)$ \\
\hline \hline$K^{+}$ & $\pi^{0}$ & $3.589 \times 10^{-6}$ & 4.45 & $3.18 \pm 0.08$ \\
$K_{S}^{0}$ & $\pi^{-}$ & $7.274 \times 10^{-6}$ & 0.0650 & $0.0670 \pm 0.0007$ \\
$K_{L}^{0}$ & $\pi^{-}$ & $7.274 \times 10^{-6}$ & 37.6 & $38.78 \pm 0.27$ \\
$D^{0}$ & $\pi^{-}$ & $5.488 \times 10^{-3}$ & 0.228 & $0.37 \pm 0.06$ \\
$D^{0}$ & $K^{-}$ & $8.476 \times 10^{-2}$ & 3.52 & $3.50 \pm 0.17$ \\
$D^{+}$ & $\pi^{0}$ & $2.790 \times 10^{-3}$ & 0.295 & $0.31 \pm 0.15$ \\
$D^{+}$ & $K^{0}$ & $8.515 \times 10^{-2}$ & 9.000 & $6.8 \pm 0.8$ \\
$D_{s}^{+}$ & $K^{0}$ & $4.184 \times 10^{-3}$ & 0.195 & \\
$D_{s}^{+}$ & $D^{0}$ & $4.786 \times 10^{-8}$ & $2.24 \times 10^{-6}$ & \\
$B^{0}$ & $\pi^{-}$ & $6.455 \times 10^{-5}$ & 0.0101 & $0.018 \pm 0.006$ \\
$B^{0}$ & $D^{-}$ & $1.716 \times 10^{-2}$ & 2.68 & $2.00 \pm 0.006$ \\
$B^{0}$ & $\left(D^{*}\right)^{-}$ & $3.983 \times 10^{-2}$ & 6.21 & $4.60 \pm 0.27$ \\
$B^{+}$ & $\pi^{0}$ & $3.457 \times 10^{-5}$ & $5.70 \times 10^{-3}$ & $<0.22$ \\
$B^{+}$ & $D^{0}$ & $1.726 \times 10^{-2}$ & 2.85 & $1.86 \pm 0.33$ \\
$B^{+}$ & $\left(D^{*}\right)^{0}$ & $4.059 \times 10^{-2}$ & 6.70 & $5.3 \pm 0.8$ \\
$B_{s}^{0}$ & $K^{-}$ & $6.118 \times 10^{-5}$ & $9.42 \times 10^{-3}$ & \\
$B_{s}^{0}$ & $D_{s}^{-}$ & $1.642 \times 10^{-2}$ & 2.53 & \\
$B_{s}^{0}$ & $\left(D_{s}^{*}\right)^{-}$ & $4.185 \times 10^{-2}$ & 6.45 & \\
$B_{s}^{0}$ & $B^{-}$ & $2.619 \times 10^{-8}$ & $4.03 \times 10^{-6}$ & \\
$B_{s}^{0}$ & $\left(B^{*}\right)^{-}$ & $8.032 \times 10^{-10}$ & $1.24 \times 10^{-7}$ & \\
& & & & \\
\hline
\end{tabular}


Table 5: Comparison of this work with other approaches for the form factor $f_{+}(0)$. Here $\mathrm{P}$ is the parent meson and $\mathrm{D}$ is the Daughter

\begin{tabular}{|c|c|c|c|c|c|c|c|c|c|}
\hline $\mathrm{P}$ & $\mathrm{D}$ & This work & {$[5]$} & {$[21]$} & {$[22$} & {$[24$} & {$[12]$} & {$[3]$} & {$[23]$} \\
\hline \hline$K^{+}$ & $\pi^{0}$ & 0.9617 & & & & & 0.98 & & \\
$D^{0}$ & $K^{-}$ & 0.7869 & 0.76 & 0.780 & 0.71 & 0.78 & 0.74 & 0.73 & 0.79 \\
$D^{0}$ & $\pi^{-}$ & 0.6292 & 0.69 & 0.681 & 0.8 & & & 0.65 & 0.86 \\
$B^{0}$ & $D^{-}$ & 0.7977 & 0.69 & 0.684 & & & 0.73 & & 0.65 \\
$B^{0}$ & $\pi^{-}$ & 0.2848 & 0.33 & 0.293 & 0.33 & 0.53 & 0.51 & 0.27 & 0.43 \\
$B_{s}^{0}$ & $K^{-}$ & 0.2452 & & & 0.36 & & & & \\
\hline
\end{tabular}

Table 6: Vector Form Factors for the Decay $B \rightarrow D^{*}+\ell^{+}+\nu_{\ell}$

\begin{tabular}{|c|c|c|c|}
\hline Reference & $g(0)\left(\mathrm{GeV}^{-1}\right)$ & $a_{+}(0)\left(\mathrm{GeV}^{-1}\right)$ & $f(0)(\mathrm{GeV})$ \\
\hline \hline This Work & -0.10391 & -0.09240 & 5.286 \\
5] & -0.09745 & -0.09471 & 4.736 \\
27 & -0.16 & -0.15 & 6.863 \\
23] & -0.09054 & -0.07271 & 3.863 \\
\hline
\end{tabular}

Table 7: Predictions for $B_{c} \rightarrow P$ Decays

\begin{tabular}{|c|c|c|c|c|}
\hline $\mathrm{P}$ & $f_{+}(0)$ & $f_{+}\left(q_{\max }^{2}\right)$ & $\Gamma\left(p s^{-1}\right)$ & $\mathrm{BR}(\%)$ \\
\hline \hline$B^{0}$ & 0.4504 & 0.6816 & $9.7001 \times 10^{-4}$ & 0.049 \\
$B_{s}^{0}$ & 0.5917 & 0.8075 & $1.8774 \times 10^{-2}$ & 0.94 \\
$D^{0}$ & 0.1446 & 1.017 & $2.8244 \times 10^{-5}$ & 0.0014 \\
$\eta_{c}$ & 0.5359 & 1.034 & $1.0355 \times 10^{-2}$ & 0.52 \\
\hline
\end{tabular}


Table 8: Predictions for $B_{c} \rightarrow V$ Decays

\begin{tabular}{|c|c|c|}
\hline $\mathrm{V}$ & $\Gamma\left(p s^{-1}\right)$ & $\mathrm{BR}(\%)$ \\
\hline \hline$\left(B^{*}\right)^{0}$ & $1.048 \times 10^{-3}$ & 0.052 \\
$\left(B_{s}^{*}\right)^{0}$ & $2.872 \times 10^{-2}$ & 1.44 \\
$\left(D^{*}\right)^{0}$ & $4.739 \times 10^{-5}$ & 0.0024 \\
$J / \psi$ & $2.943 \times 10^{-2}$ & 1.47 \\
\hline
\end{tabular}

Table 9: Predictions for the form factors at $q^{2}=0$ for $B_{c} \rightarrow V$ decays.

\begin{tabular}{|c|c|c|c|}
\hline $\mathrm{V}$ & $g(0) \mathrm{GeV}^{-1}$ & $a_{+}(0) \mathrm{GeV}^{-1}$ & $f(0) \mathrm{GeV}$ \\
\hline \hline$\left(B^{*}\right)^{0}$ & -0.1671 & -0.0463 & 3.383 \\
$\left(B_{s}^{*}\right)^{0}$ & -0.2402 & -0.0673 & 5.506 \\
$\left(D^{*}\right)^{0}$ & -0.0211 & -0.0127 & 0.8296 \\
$J / \psi$ & -0.0784 & -0.0543 & 4.918 \\
\hline
\end{tabular}

Table 10: Branching Ratios for the Semileptonic Decays of the $B_{c}$.

\begin{tabular}{|c|c|c|c|c|c|}
\hline Decay Meson & This Work & [4] & [28] & [29] & [30] \\
\hline \hline$B_{s}^{0}$ & $0.94 \%$ & $(0.68 \pm 0.23) \%$ & $1.35 \%$ & & $0.80 \%$ \\
$\left(B_{s}^{*}\right)^{0}$ & $1.44 \%$ & $(0.68 \pm 0.23) \%$ & $3.22 \%$ & & $2.3 \%$ \\
$\eta_{c}$ & $0.52 \%$ & $(0.57 \pm 0.17) \%$ & $0.553 \%$ & $0.836 \%$ & $0.15 \%$ \\
$J / \psi$ & $1.47 \%$ & $(0.68 \pm 0.17) \%$ & $2.32 \%$ & $2.13 \%$ & $1.5 \%$ \\
\hline
\end{tabular}


Table 11: The decay constant $g_{V P \gamma}$ in $\mathrm{GeV}^{-1}$.

\begin{tabular}{|c|c|c|c|c|c|c|}
\hline $\mathrm{V}$ & This Work & Expt. 14 & {$[23$} & 32] & 33] & 13 \\
\hline \hline$\left(D^{*}\right)^{0}$ & 2.0321 & & 1.043 & 1.079 & 1.598 & 1.939 \\
$\left(D^{*}\right)^{+}$ & 0.5224 & & 0.1535 & 0.0702 & 0.2886 & 0.3950 \\
$\left(D_{s}^{*}\right)^{+}$ & 0.2369 & & & & 0.1917 & 0.2598 \\
$J / \psi$ & 0.7419 & 0.5538 & & & & \\
$\left(B^{*}\right)^{0}$ & 0.9770 & & 0.3098 & 0.4177 & 0.5720 & 0.9104 \\
$\left(B^{*}\right)^{+}$ & 1.7627 & & 0.3461 & 0.6540 & 0.9701 & 1.618 \\
$\left(B_{s}^{*}\right)^{0}$ & 0.6417 & & & & & \\
$\Upsilon$ & 0.1314 & & & & & \\
\hline
\end{tabular}

Table 12: Calculations of $B_{c}^{*}$ Properties and $g_{B_{c}^{*} B_{c} \gamma}$.

\begin{tabular}{|c|c|c|c|}
\hline$M_{B_{c}^{*}}(\mathrm{GeV})$ & $\Pi\left(\left(M_{B_{c}^{*}}\right)^{2}\right)\left(\mathrm{GeV}^{2}\right)$ & $g_{B_{c}^{*}}$ & $g_{B_{c}^{*} B_{c} \gamma}\left(\mathrm{GeV}^{-1}\right)$ \\
\hline \hline 6.25 & 0.2843 & 8.627 & 0.3013 \\
6.26 & 0.2877 & 8.541 & 0.3011 \\
6.27 & 0.2912 & 8.455 & 0.3008 \\
6.28 & 0.2947 & 8.369 & 0.3006 \\
6.29 & 0.2984 & 8.283 & 0.3003 \\
6.30 & 0.3021 & 8.196 & 0.3001 \\
6.31 & 0.3059 & 8.109 & 0.2999 \\
6.32 & 0.3097 & 8.022 & 0.2995 \\
6.33 & 0.3137 & 7.934 & 0.2993 \\
6.34 & 0.3178 & 7.846 & 0.2990 \\
6.35 & 0.3220 & 7.758 & 0.2987 \\
\hline
\end{tabular}




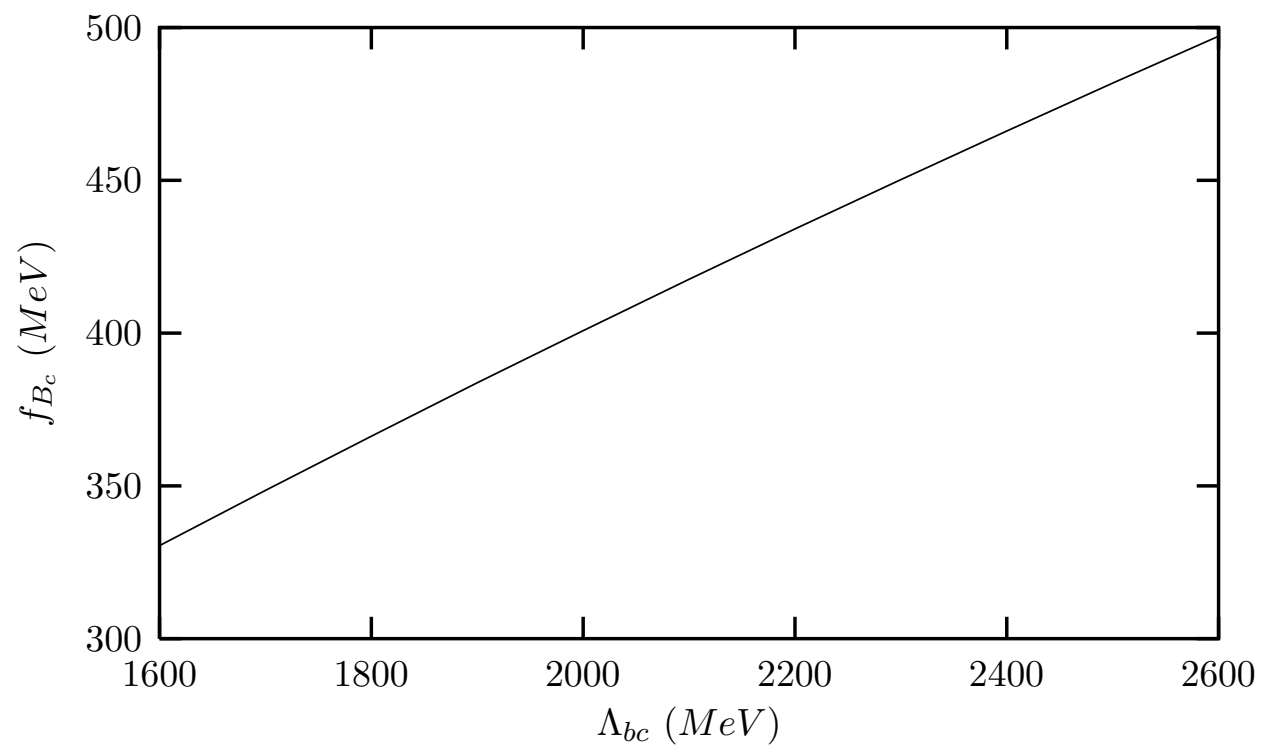

Figure 1: $f_{B_{c}}$ vs. $\Lambda_{b c}$ 


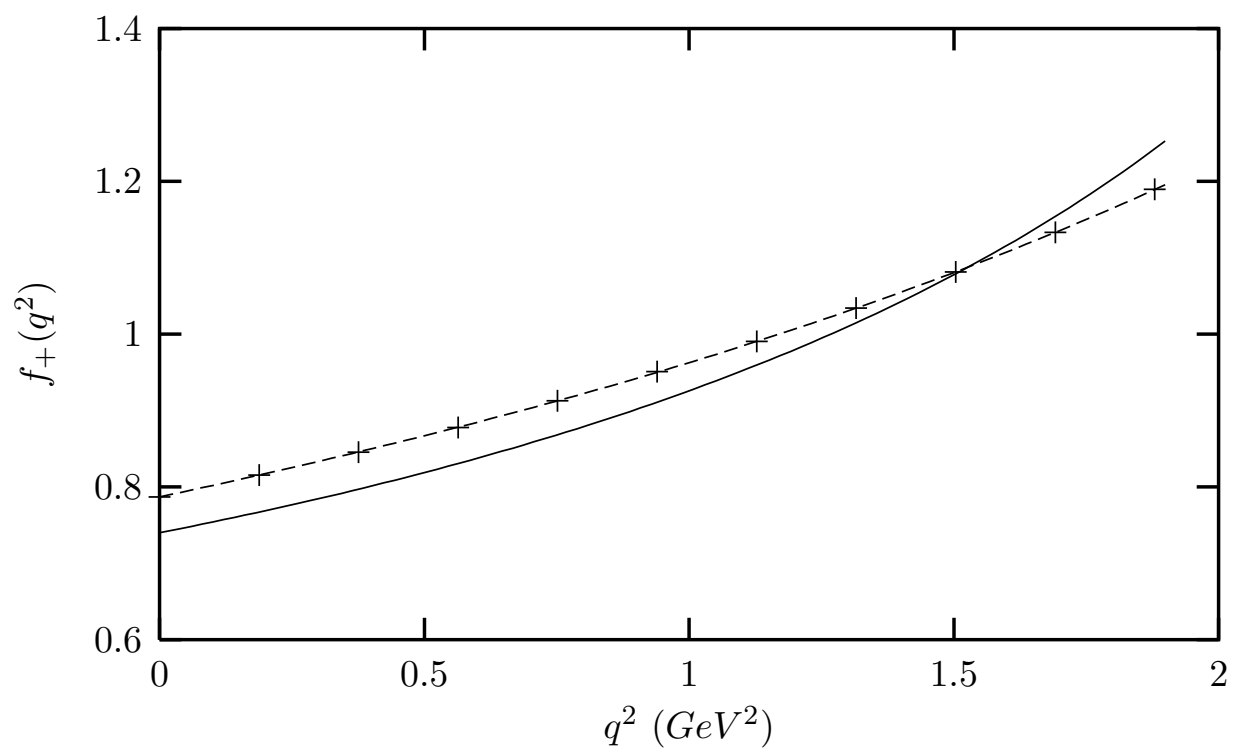

Figure 2: Form factor for the decay $D^{0} \rightarrow K^{-}$calculated in this work (dashed line) and in Ref. [12] (solid line). 


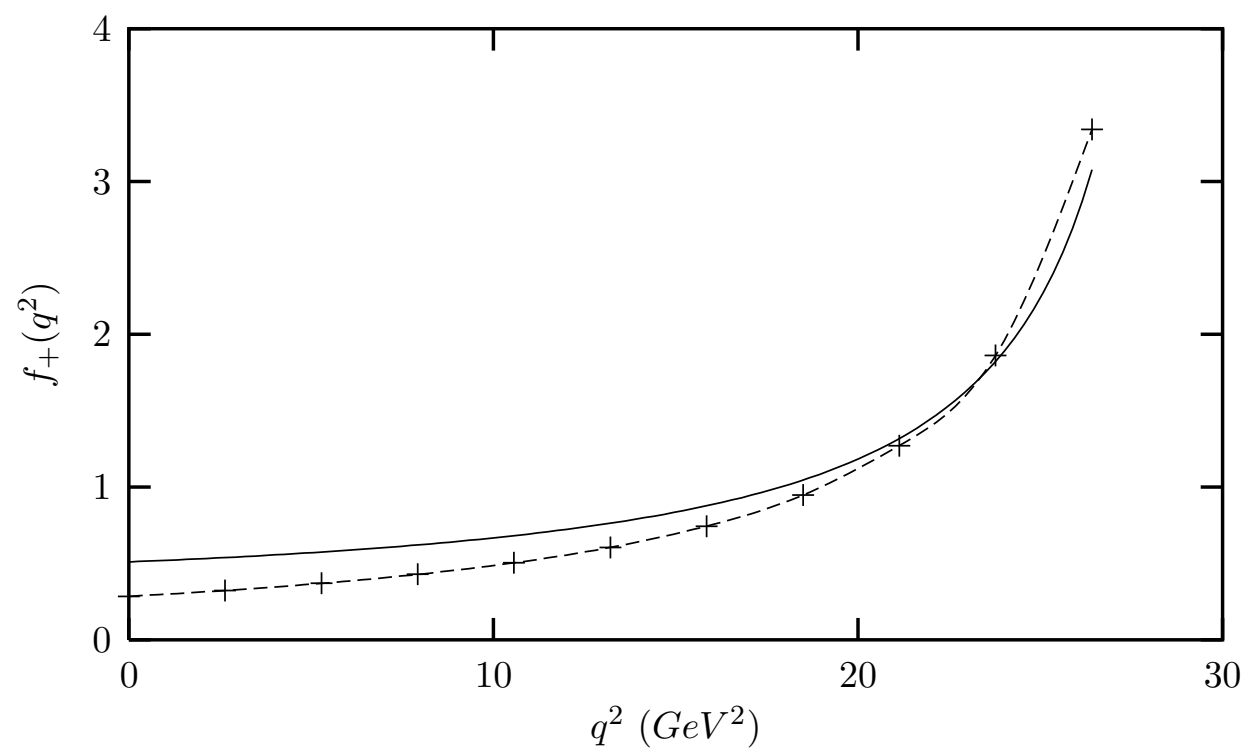

Figure 3: Form factor for the decay $B^{0} \rightarrow \pi^{-}$calculated in this work (dashed line) and in Ref. [12] (solid line).

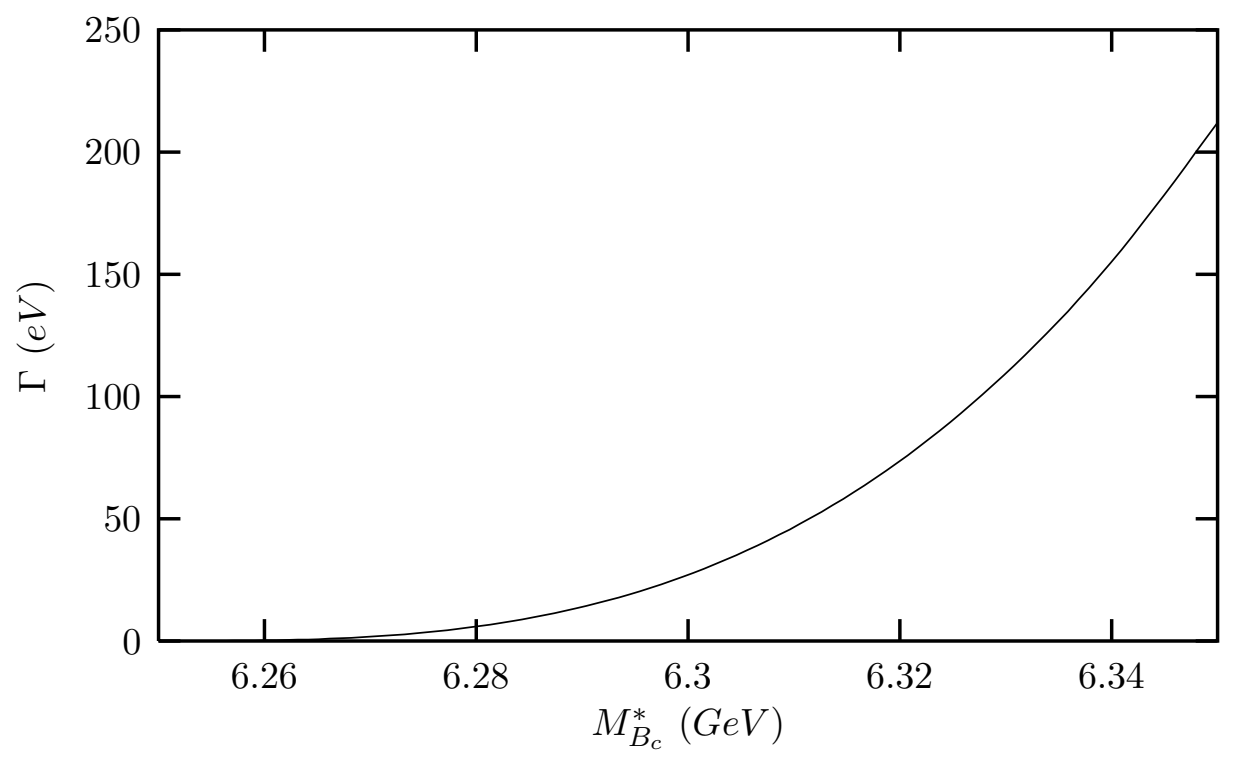

Figure 4: The decay rate $\Gamma\left[B_{c}^{*} \rightarrow B_{c}+\gamma\right]$ vs. $M_{B_{c}^{*}}$. 